\title{
Benign Hepatic Nodules Mimicking Hepatocellular Carcinoma in the Setting of Fontan-associated Liver Disease: A Case Report
}

\author{
Anıl Çolaklar ${ }^{1}$, Stephen J Lehnert ${ }^{2}$, Temel Tirkes $^{3}$
}

\begin{abstract}
Fontan procedure, in which systemic circulation is redirected into pulmonary circulation by a baffle, is a palliative surgical strategy for patients born with single ventricle congenital heart disease. Hemodynamic changes secondary to Fontan procedure, also termed as Fontan physiology, result in end-organ damage, especially of the liver. Fontan-associated liver disease (FALD) represents a spectrum of pathologies ranging from mild liver fibrosis to advanced liver cirrhosis and hepatocellular carcinoma (HCC). Hepatic nodules, some of which have been documented as HCC in several case series and reports, are a recognized complicated feature of FALD. Herein, we report a case with benign hepatic nodules mimicking $\mathrm{HCC}$ by imaging characteristics, emphasizing the fact that arterially enhancing lesions with delayed washout appearance may reflect benign regenerative or focal nodular hyperplasia-like nodules in patients with Fontan physiology.

Keywords: Congenital heart disease, Fontan procedure, Imaging features, Liver lesion.

Euroasian Journal of Hepato-Gastroenterology (2020): 10.5005/jp-journals-10018-1315
\end{abstract}

\section{INTRODUCTION}

Fontan procedure, which was originally introduced in 1971 by Fontan and Baudet, ${ }^{1}$ is a palliative surgical operation for patients born with single ventricle congenital heart disease. This strategy redirects systemic venous blood flow directly to pulmonary circulation to minimize the oxygenated-deoxygenated blood mixture and alleviate the burden on a single ventricle, ${ }^{1}$ which consequently results in elevated central venous pressure and diminished cardiac output. ${ }^{2}$ These hemodynamic changes, also termed as Fontan physiology, cause end-organ damage throughout the body and thus present with some debilitating pathologies, such as peripheral venous insufficiency, plastic bronchitis, protein-losing enteropathy, and Fontan-associated liver disease (FALD).,

We present a case of FALD in an adult with histopathologically proven benign nodules mimicking hepatocellular carcinoma (HCC) by imaging criteria. Arterially enhancing benign hepatic nodules with a washout appearance on both portal and more delayed venous phase images are rare, with very few scattered case reports throughout the English literature to date. ${ }^{5}$ Large-scale studies are needed to overcome the limitations of rare case reports.

\section{Case Description}

A 37-year-old woman was referred to our ultrasonography (US) department with right upper quadrant pain. She had a history of pulmonary atresia with complex univentricular anatomy. She underwent five aortopulmonary shunt operations in early childhood, followed by classic Fontan operation at ten years of age (Fig. 1). She was on amiodarone and aspirin for atrial flutter. The blood test results on admission were unremarkable. Hepatitis $B$ and $C$ virus serologies were negative, and there was no history of alcohol abuse. She had not been under any hepatic surveillance and had not undergone any hepatic imaging previously. Physical examination revealed abdominal tenderness in epigastrium and right upper quadrant with palpation.
${ }^{1-3}$ Department of Radiology and Imaging Sciences, Indiana University School of Medicine, Indianapolis, Indiana, USA

Address reprint requests to: Anıl Çolaklar, Department of Radiology and Imaging Sciences, Indiana University School of Medicine, Indianapolis, Indiana, USA, Phone: +905432026537, e-mail: anilcolaklar@gmail.com

How to cite this article: Çolaklar A, Lehnert SJ, Tirkes T. Benign Hepatic Nodules Mimicking Hepatocellular Carcinoma in the Setting of Fontan-associated Liver Disease: A Case Report. Euroasian J HepatoGastroenterol 2020;10(1):42-44.

Source of support: National Cancer Institute and National Institute of Diabetes and Digestive and Kidney Diseases of the National Institutes of Health

Conflict of interest: None

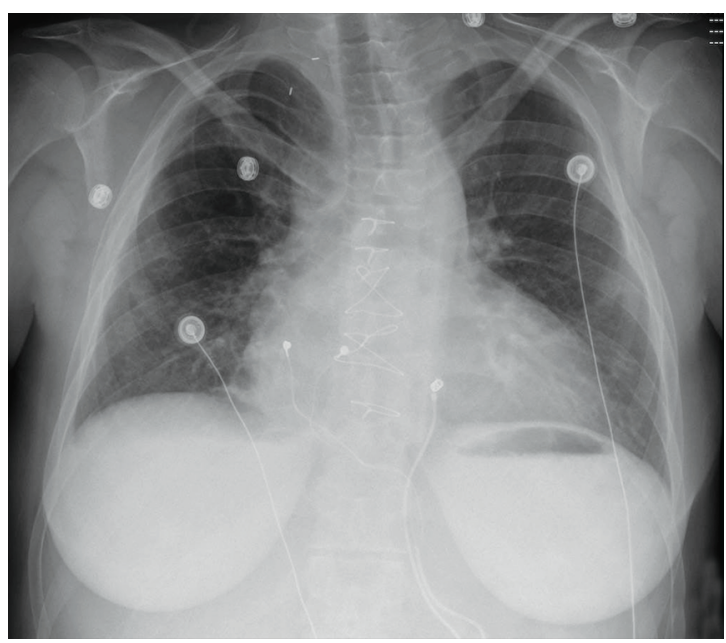

Fig. 1: Anteroposterior chest radiograph shows a median sternotomy defect due to previous open-heart surgery and mild cardiomegaly with epicardial pacer/leads 
Grayscale and Doppler US examinations showed cirrhotic features, consistent with FALD. Additionally, several hypervascular liver lesions were found with a dominant lesion in segment 7 concerning HCC in the cirrhotic background (Fig. 2). However, serum alpha-fetoprotein level was within the normal range.
Triphasic computed tomography (CT) of the liver was subsequently performed to further evaluate these findings on US. The lesion in question showed enhancement during the arterial phase, followed by washout during the portal and late venous phases (Fig. 3). Given the past medical history and radiological findings, US-guided
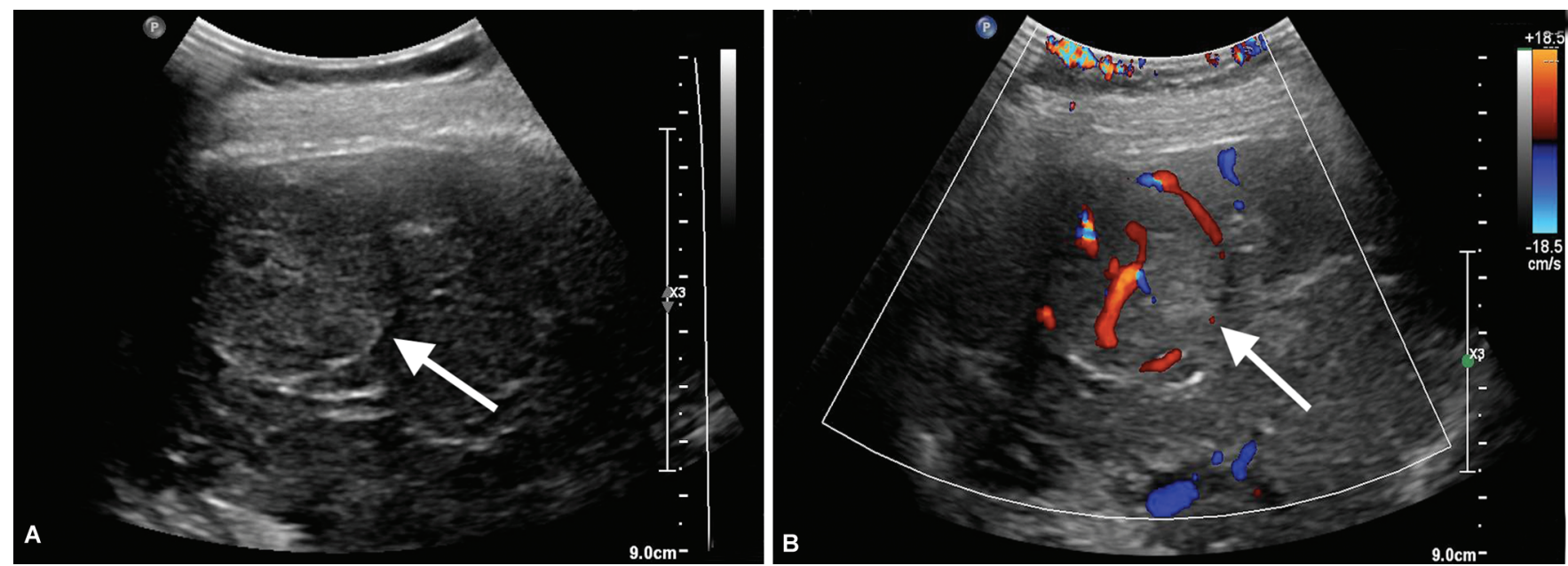

Figs 2A and B: Grayscale (A) and color Doppler (B) US images demonstrate a well-circumscribed, hypervascular hepatic nodule (arrows) in segment 7
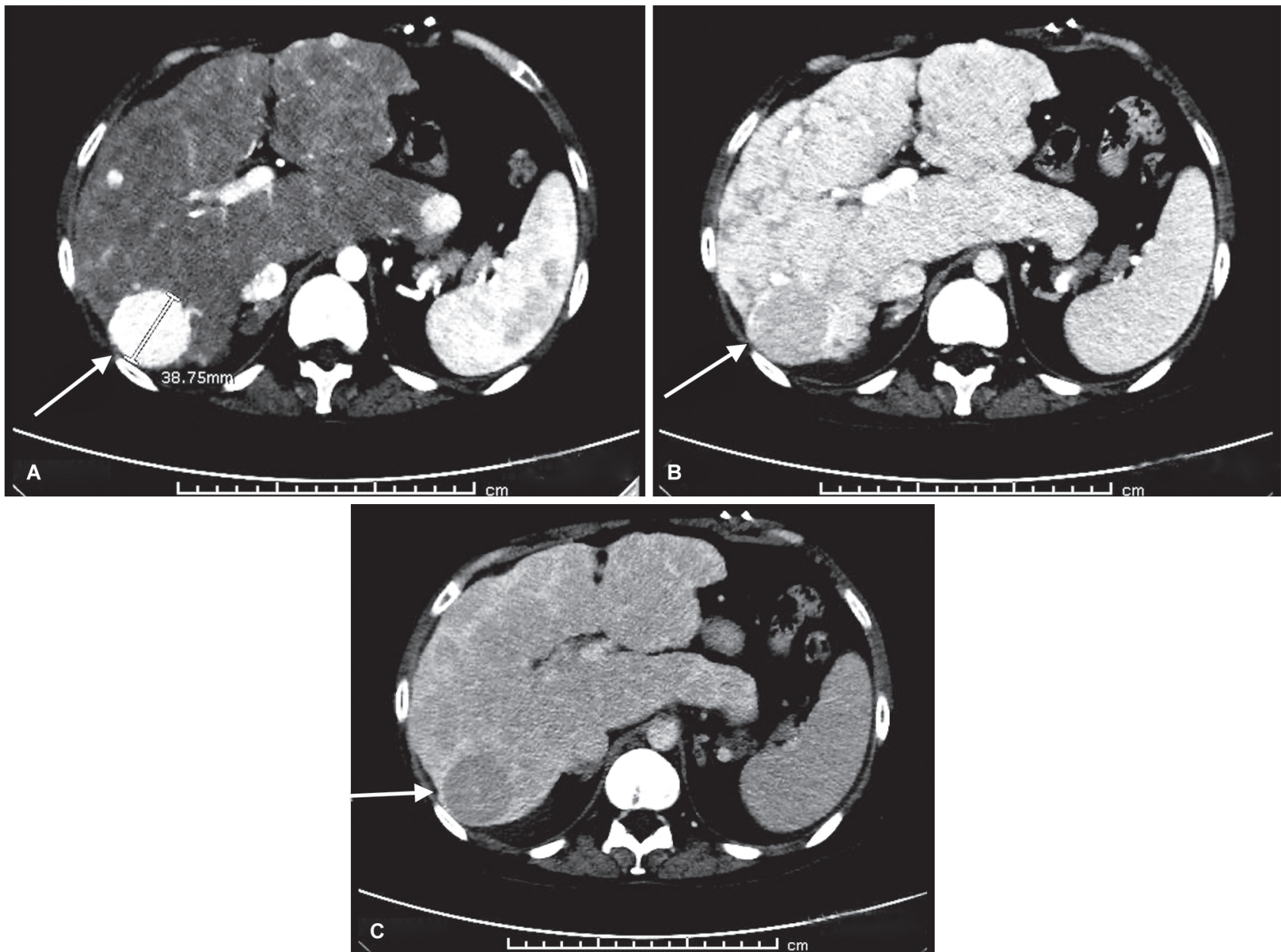

Figs $3 \mathrm{~A}$ to $\mathrm{C}$ : Axial contrast-enhanced CT images reveal avid enhancement of the dominant nodule (arrows) during the late arterial phase (A) with a washout appearance during the portal $(B)$ and more delayed $(C)$ venous phases. Note that other arterially enhancing liver nodules do not show any absolute washout on delayed phase images 
biopsy was performed from the dominant lesion. The biopsy revealed sinusoidal dilatation and focal fibrosis without any features concerning malignancy. On follow-up examination, hepatic lesions showed size stability over 12 months, and thus benign etiology was favored. At the time of this case report, the patient is doing well on a routine follow-up program for FALD.

\section{Discussion}

Fontan-associated liver disease refers to a spectrum of pathologies of the liver secondary to hemodynamic changes associated with Fontan physiology. Elevated central venous pressure and diminished cardiac output lead to hepatic venous congestion, ischemia, and eventually fibrosis. ${ }^{6}$ The histological features in the liver of patients with Fontan physiology are similar to those seen in congestive heart failure. ${ }^{7}$ Histological studies also show that almost half of the patients have developed advanced liver cirrhosis within 30 years after the Fontan procedure. ${ }^{8}$ In most of the patients with Fontan physiology, liver fibrosis is further complicated by hepatic nodules, some of which were proved to be HCC throughout the literature..$^{3-5,9-18}$ However, arterially enhancing benign hepatic nodules in this patient population may demonstrate washout on portal venous and/or more delayed phase images, as in our case. ${ }^{5}$ Hence, not all arterially enhancing nodules with a washout appearance on portal venous and/or more delayed phase images can be considered malignant. Although the underlying mechanism has not yet been elucidated clearly, these findings are postulated to be secondary to hemodynamic changes associated with Fontan physiology. Radiologists should consider histological confirmation before making the definitive diagnosis of HCC by imaging criteria.

\section{Conclusion}

Fontan-associated liver disease is an inevitable consequence of the Fontan procedure. In this patient population, arterially enhancing benign hepatic nodules with delayed washout pose a diagnostic challenge since these enhancement features overlap with HCC. Both radiologists and hepatologists should be aware of this benign complication not to misdiagnose these nodules as HCC. In FALD patients, all arterially enhancing hepatic nodules with delayed washout should be approached with caution, which may require follow-up imaging to document stability or biopsy if clinically indicated.

\section{Ethics Committee Approval}

The Institutional Review Board (IRB) approved this study.

\section{Informed Consent}

The requirement for the informed consent was waived since all the content herein has been fully deidentified.

\section{Financial Disclosure}

This research received no direct funding. However, Dr Tirkes is supported by the National Cancer Institute and National Institute of Diabetes and Digestive and Kidney Diseases of the National Institutes of Health under award numbers 1R01DK116963 and U01DK108323 (Consortium for the Study of Chronic Pancreatitis, Diabetes, and Pancreatic Cancer). The content is solely the responsibility of the authors and does not necessarily represent the official views of the National Institutes of Health.

\section{Note}

All the contents herein have been fully deidentified to protect the patient's privacy.

\section{References}

1. Fontan F, Baudet E. Surgical repair of tricuspid atresia. Thorax 1971;26(3):240-248. DOI: 10.1136/thx.26.3.240.

2. Davies RR, Chen JM, Mosca RS. The Fontan procedure: evolution in technique; attendant imperfections and transplantation for "failure". Semin Thorac Cardiovasc Surg Pediatr Card Surg Annu 2011;14(1):55-66. DOI: 10.1053/j.pcsu.2011.01.014.

3. Gordon-Walker TT, Bove K, Veldtman G. Fontan-associated liver disease: A review. J Cardiol 2019;74(3):223-232. DOI: 10.1016/ j.jjcc.2019.02.016.

4. Tellez $L$, Rodriguez de Santiago $E$, Albillos A. Fontan-associated liver disease. . Rev Esp Cardiol (Engl Ed) 2018;71(3):192-202. DOI: 10.1016/ j.recesp.2017.10.014.

5. Wells ML, Hough DM, Fidler JL, et al. Benign nodules in postFontan livers can show imaging features considered diagnostic for hepatocellular carcinoma. Abdom Radiol (NY) 2017;42(11):2623-2631. DOI: 10.1007/s00261-017-1181-9.

6. Asrani SK, Asrani NS, Freese DK, et al. Congenital heart disease and the liver. Hepatology 2012;56(3):1160-1169. DOI: 10.1002/hep.25692.

7. Kendall TJ, Stedman B, Hacking N, et al. Hepatic fibrosis and cirrhosis in the Fontan circulation: a detailed morphological study. J Clin Pathol 2008;61(4):504-508. DOI: 10.1136/jcp.2007.052365.

8. Pundi K, Pundi KN, Kamath PS, et al. Liver disease in patients after the Fontan operation. Am J Cardiol 2016;117(3):456-460. DOI: 10.1016/j. amjcard.2015.11.014.

9. Ghaferi AA, Hutchins GM. Progression of liver pathology in patients undergoing the Fontan procedure: Chronic passive congestion, cardiac cirrhosis, hepatic adenoma, and hepatocellular carcinoma. J Thorac Cardiovasc Surg 2005;129(6):1348-1352. DOI: 10.1016/j. jtcvs.2004.10.005.

10. Asrani SK, Warnes CA, Kamath PS. Hepatocellular carcinoma after the Fontan procedure. N Engl J Med 2013;368(18):1756-1757. DOI: 10.1056/NEJMc1214222.

11. Elder RW, Parekh S, Book WM. More on hepatocellular carcinoma after the Fontan procedure. N Engl J Med 2013;369(5):490. DOI: 10.1056/ NEJMc1306854.

12. Kwon S, Scovel L, Yeh M, et al. Surgical management of hepatocellular carcinoma after Fontan procedure. J Gastrointest Oncol 2015;6(3): E55-E60.

13. Yamada K, Shinmoto H, Kawamura Y, et al. Transarterial embolization for pediatric hepatocellular carcinoma with cardiac cirrhosis. Pediatr Int 2015;57(4):766-770. DOI: 10.1111/ped.12619.

14. Takuma Y, Fukada Y, Iwadou S, et al. Surgical resection for hepatocellular carcinoma with cardiac cirrhosis after the Fontan procedure. Intern Med. 2016;55(22):3265-3272. DOI: 10.2169/ internalmedicine.55.6869.

15. Oh C, Youn JK, Han JW, et al. Hepatocellular carcinoma after the Fontan procedure in a 16-year-old girl: A case report. Medicine (Baltimore) 2016;95(41):e4823. DOI: 10.1097/MD.0000000000004823.

16. Josephus Jitta D, Wagenaar LJ, Mulder BJ, et al. Three cases of hepatocellular carcinoma in Fontan patients: Review of the literature and suggestions for hepatic screening. Int J Cardiol 2016;206:21-26. DOI: 10.1016/j.ijcard.2015.12.033.

17. Conroy MR, Moe TG. Hepatocellular carcinoma in the adult Fontan patient. Cardiol Young. 2017;27(2):407-409. DOI: 10.1017/ S1047951116001360.

18. Martinez-Quintana E, Monescillo A, Rodriguez-Gonzalez F. Hepatocellular carcinoma in a non-failing Fontan circulation. Rev Esp Enferm Dig 2017;109(5):375. 